\title{
Invasive Sphenocavernous Aspergilloma Complicating an Operated Case of Acromegaly
}

\author{
Sunil V. Furtado, M.S., M.Ch., D.N.B., ${ }^{1}$ Prasanna K. Venkatesh, D.N.B., ${ }^{1}$ \\ Nandita Ghosal, M.D., ${ }^{2}$ and Alangar S. Hegde, M.Ch., Ph.D. ${ }^{1}$
}

There is a surge in fungal infections of the central nervous system due to an increase in the incidence of immunocompromised state and widespread use of antimicrobials. Despite myriad clinical presentations and skull base syndromes associated with Aspergillus infections, aspergilloma in the sella is relatively rare. We report a rare case of aspergilloma of the sella and parasellar region in a patient operated for acromegaly. A 30-year-old diabetic patient underwent two surgeries for a growth hormone-secreting pituitary adenoma, which included a transsphenoidal approach. During his last admission, magnetic resonance imaging revealed a heterogeneous mass in the sella, suprasellar region, sphenoid sinus, and cavernous sinus with multiple areas of necrosis. His presentation, surgical treatment, and response to antifungal therapy are discussed. The role of prior surgery and related comorbidities in developing the invasive granuloma are highlighted.

KEYWORDS: Acromegaly, amphotericin, Aspergillus, cavernous sinus, fungal infections, sella, surgery

\begin{abstract}
Aspergillus species that are present in soil and decaying matter enter the body through the respiratory tract and paranasal sinuses and cause granulomas in the paranasal sinus and central nervous system $(\mathrm{CNS}) .{ }^{1,2}$ Dual pathologies associated with granulomatous infections within the sella are extremely rare, and only three cases have been reported earlier, of which two were tuberculomas associated with acromegaly and one with a prolactinoma. ${ }^{3,4}$ There are five previous reports of sellar aspergillosis in indexed literature, most of them being pituitary abscesses. ${ }^{5-8}$ However, these were not associated with a pituitary tumor. Fungal granulomas are known to mimic pituitary adenomas especially when associated with an atypical presentation and previous
\end{abstract}

history of sellar surgery and are known to have a high morbidity and mortality. ${ }^{9}$ Our case illustrates a diagnostic dilemma in a diabetic patient operated for acromegaly who presented in an obtunded state.

\section{CASE REPORT}

A 30-year-old male diabetic patient presented in 2003 with a history of reduced visual acuity, headache, and morphological features of acromegaly. Magnetic resonance imaging (MRI) revealed a sellar, suprasellar contrast-enhancing mass suggestive of pituitary adenoma (Figs. 1a and 1c). In view of the large size of the lesion, the patient underwent a pterional craniotomy and
Departments of ${ }^{1}$ Neurosurgery and ${ }^{2}$ Pathology, Sri Sathya Sai Institute of Higher Medical Sciences, EPIP Area, Karnataka State, Bangalore, India.

Address for correspondence and reprint requests: Sunil V. Furtado, M.S., M.Ch., D.N.B., Department of Neurosurgery, SSSIHMS, EPIP Area, Whitefield, Bangalore-560066, India (e-mail: sunilvf@ gmail.com).
Skull Base Rep 2011;1:33-38. Copyright (C) 2011 by Thieme Medical Publishers, Inc., 333 Seventh Avenue, New York, NY 10001, USA. Tel: +1 (212) 584-4662.

Received: August 10, 2010. Accepted: November 23, 2010. Published online: March 16, 2011.

DOI: http://dx.doi.org/10.1055/s-0031-1275254.

ISSN 2157-6971. 


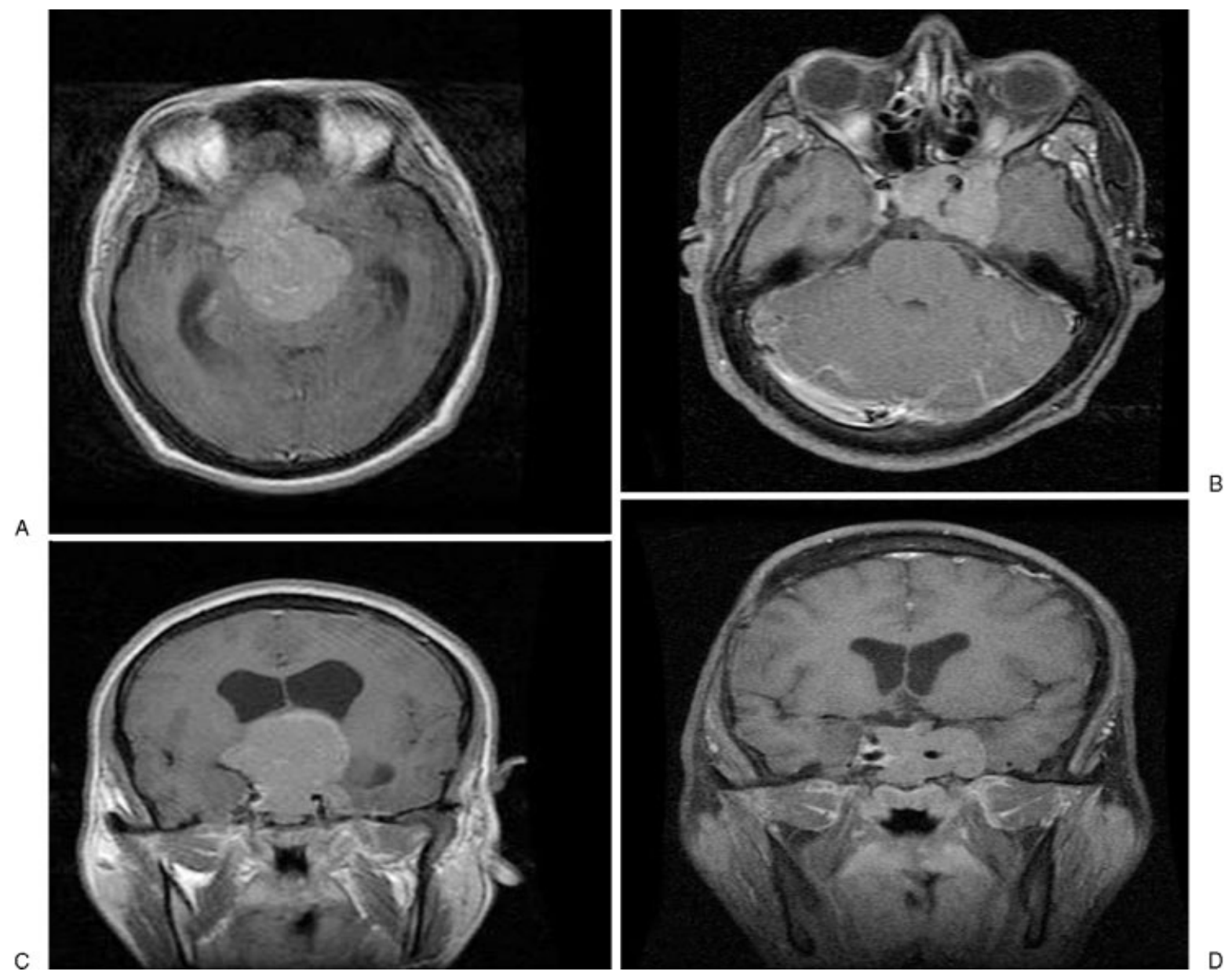

Figure 1 (A) Axial and (C) coronal contrast images of the pituitary adenoma in 2003; (B) axial and (D) coronal contrast images in 2005.

subtotal decompression of the tumor. He had an uneventful postoperative period and was discharged on antiepileptics and 1-thyroxin. He was readmitted after 18 months for surgery of the residual tumor (Fig. $1 \mathrm{~b}$ and $1 \mathrm{~d})$, and this time a transnasal transsphenoidal approach was preferred. He was advised radiotherapy for the residual lesion, but defaulted on the same.

He presented in an obtunded state in August 2010, was hypothermic, and had left-sided ophthalmoplegia with bilateral ptosis. Laboratory examination revealed a high erythrocyte sedimentation rate of $100 \mathrm{~mm} / \mathrm{h}$, and he was in diabetic ketoacidosis. Hormonal assay revealed panhypopituitarism. His hormone assays at the three admissions are presented in Table 1 MRI revealed a $4 \times 3.5 \times 3-\mathrm{cm}$ heterogeneous mass in the sellar-suprasellar region, sphenoid sinus, and cavernous sinus with multiple areas of necrosis and a thin rim of contrast enhancement (Fig. 2). Computed tomography (CT) demonstrated erosion of the sellar floor and sphenoid sinus wall (Fig. 3a). Magnetic resonance spectroscopy (MRS) revealed large lipid-lactate peak with decreased $\mathrm{N}$-acetylaspartate and choline inside the lesion (Fig. 3b). He received injectable steroids (hydrocortisone $100 \mathrm{mg}$ thrice daily) and 1-thyroxin (oral, $0.1 \mathrm{mg}$ per day) and underwent emergency external ventricular drainage for hydrocephalus. The cerebrospinal fluid (CSF) micro-

\section{Table 1 Hormonal Assay of the Patient}

\begin{tabular}{|c|c|c|c|c|}
\hline Hormones & Normal Range & 2003 & 2005 & 2009 \\
\hline Total T3 (thyronine), ng/mL & $0.8-1.7$ & 0.71 & 1.23 & 0.15 \\
\hline Total T4 (thyroxine), $\mu \mathrm{g} / \mathrm{dL}$ & $6-12$ & 4.58 & 10.28 & 2.89 \\
\hline Thyroid-stimulating hormone $(\mu \mathrm{lU} / \mathrm{mL})$ & $0.3-5$ & 0.17 & 0.14 & 0.09 \\
\hline Prolactin $(\mathrm{ng} / \mathrm{mL})$ & $3-26$ & 16.05 & 12.2 & 4.2 \\
\hline Morning serum cortisol ( $\mu \mathrm{g} / \mathrm{dL})$ & $8-22$ & 17.2 & 10.77 & 1.35 \\
\hline Growth hormone (ng/mL) & $0.004-1.406$ & 21.9 & 9.1 & 1.6 \\
\hline
\end{tabular}


A
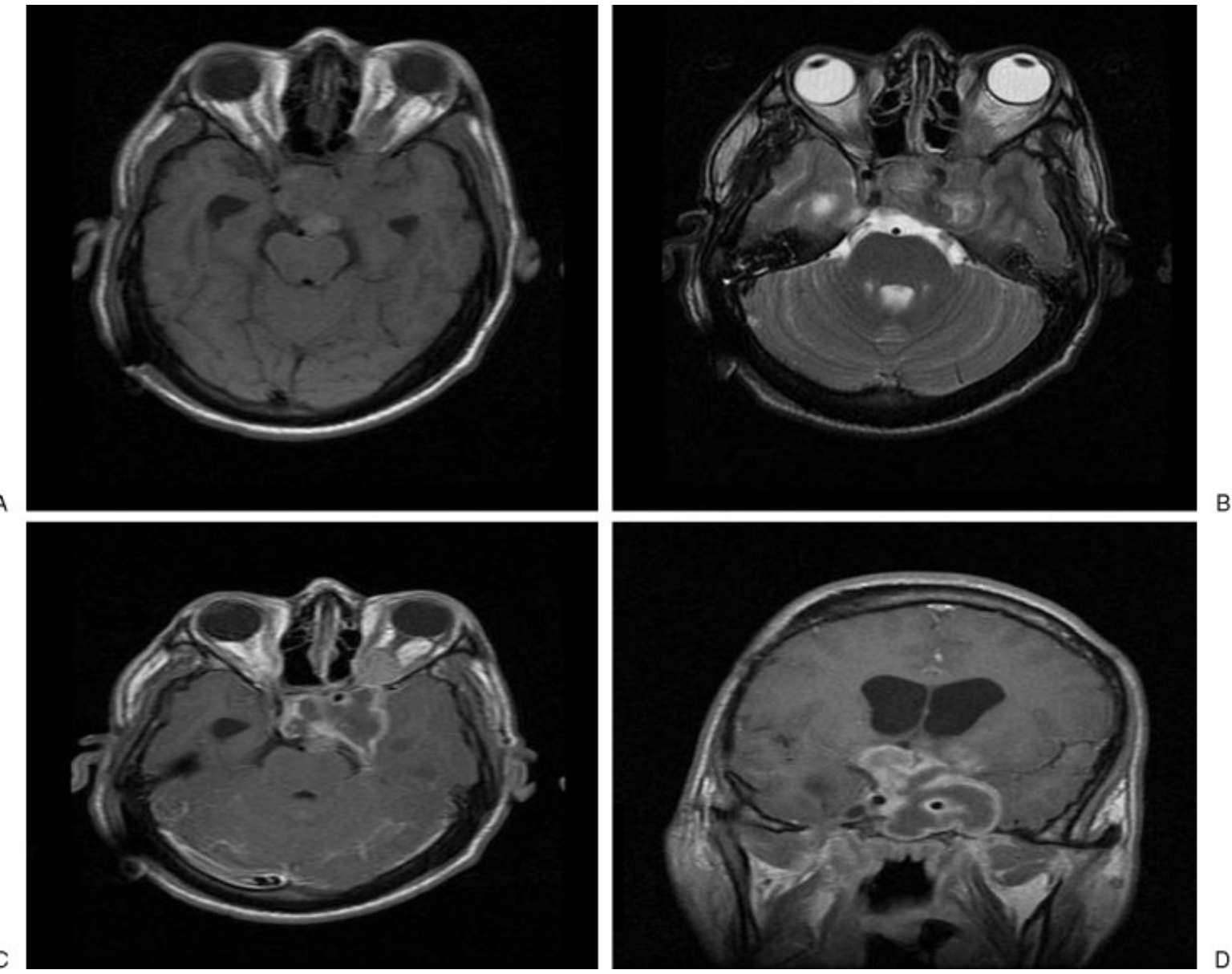

Figure 2 (A) Axial T1-weighted images showing an isointense lesion in the sella and cavernous sinus. (B) Lesion is iso- to hyperintense on axial T2-weighted images. (C) Axial and (D) coronal contrast images showing peripheral contrast enhancement of lesion extending into the sphenoid sinus, cavernous sinus, and suprasellar region.

biology was sterile for bacteria and fungi, and the external drainage was subsequently converted into a ventriculoperitoneal shunt.

He then underwent transnasal decompression of the lesion. The anatomy of the sphenoid sinus and sella was distorted. The lesion was avascular and firm, and the pituitary gland could not be identified separately. Histopathology demonstrated fungal hyphae within necrotizing infiltrates with septa of Aspergillus seen on Gomori's methenamine silver staining (Fig. 3c). Fungal culture of the material grew Aspergillus fumigatus. Postoperatively, he received amphotericin-B, 25 $\mathrm{mg} / \mathrm{d}$ for 2 weeks followed by $50 \mathrm{mg} / \mathrm{d}$ for 3 weeks (cumulative dose of $1.4 \mathrm{~g}$ ). Though there was a remarkable improvement in his sensorium, the ophthalmoplegia did not recover. He was discharged on oral steroids (tablet hydrocortisone $25 \mathrm{mg}$ per day) and 1thyroxin (oral, $0.1 \mathrm{mg}$ per day) and oral fluconazole 300 mg per day for 1 month. An MRI scan done at followup after 7 months showed near total resolution of the fungal granuloma with a small sphenoid sinus residual lesion (Fig. 4).

\section{DISCUSSION}

Fungal infections of the CNS were very rare before the advent of immunosuppressant drugs, organ transplantation, and HIV disease. ${ }^{5,6,10}$ This is highlighted by the fact that fewer than 10 cases of CNS fungal infections were identified by Keye and Magee on reviewing 15,000 autopsies from 1919 to $1955 .{ }^{10}$ The typical route of entry of Aspergillus is through inhalation of spores, where the host's normal response in the respiratory tree is a hypersensitivity reaction. ${ }^{2,10}$ Dubey et al have reported that diabetes was the most immunocompromising factor present in $40 \%$ of reported patients with CNS fungal infections. ${ }^{5}$ A reduction in cell-mediated immunity underlies CNS aspergillosis, which can present as meningitis, encephalitis, brain abscess, mycotic aneurysm, subdural abscess, or sellar abscess. ${ }^{5,6,10}$ The route of infection may be through blood, contiguous spread from paranasal sinuses, or direct inoculation of the pathogen from neurosurgical procedures. ${ }^{6,11}$ Our patient was diabetic and had undergone transsphenoidal surgery for acromegaly, which enhanced the spread and growth of the fungal granuloma in the anterior skull base. 

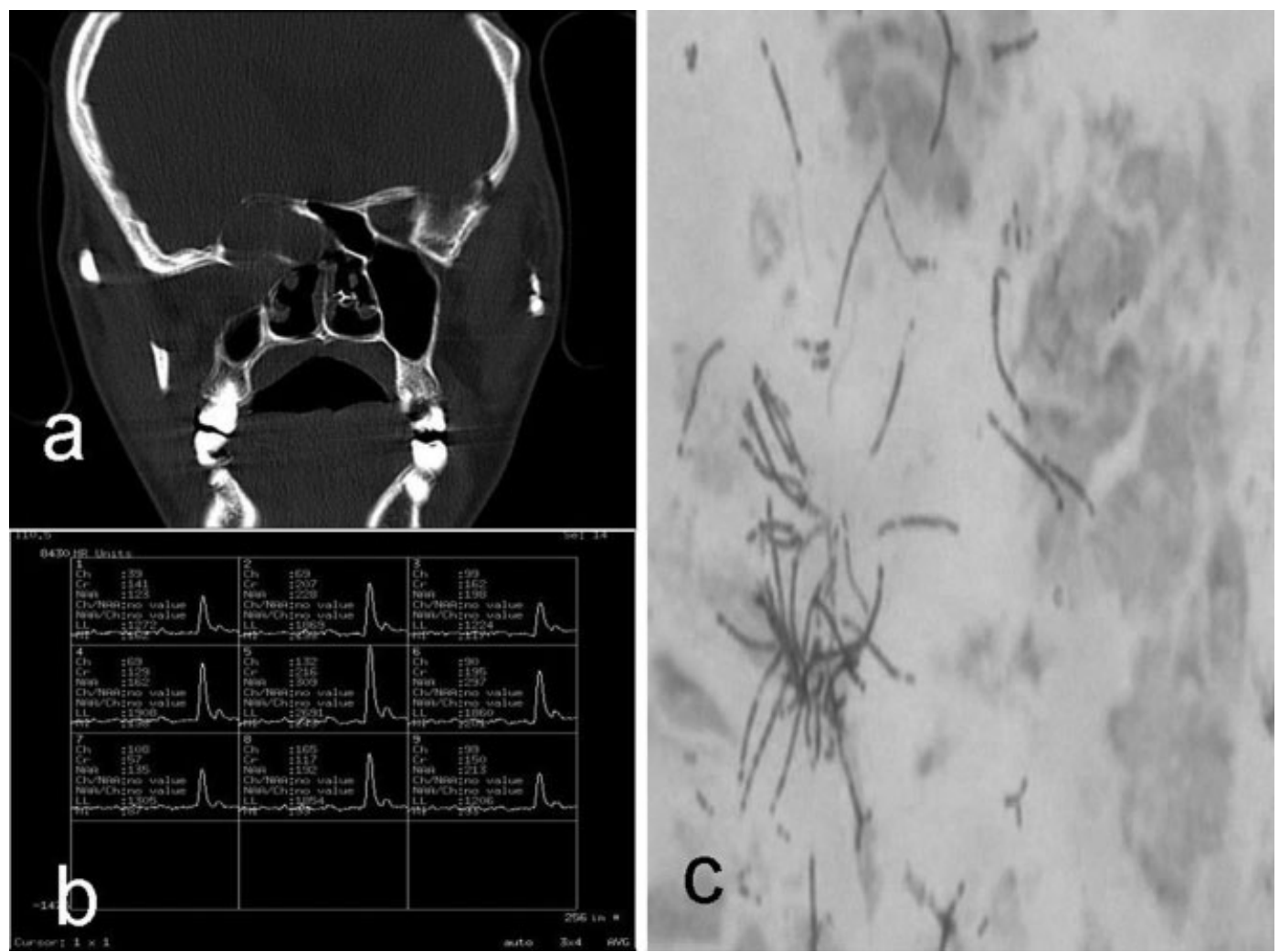

Figure 3 (a) Coronal computed tomography bone window showing erosion of the sphenoid sinus wall on the right side. (b) Magnetic resonance spectroscopy demonstrating high lactate peaks. (c) Septate fungal hyphae seen on Gomori's methenamine silver staining $(\times 400)$.
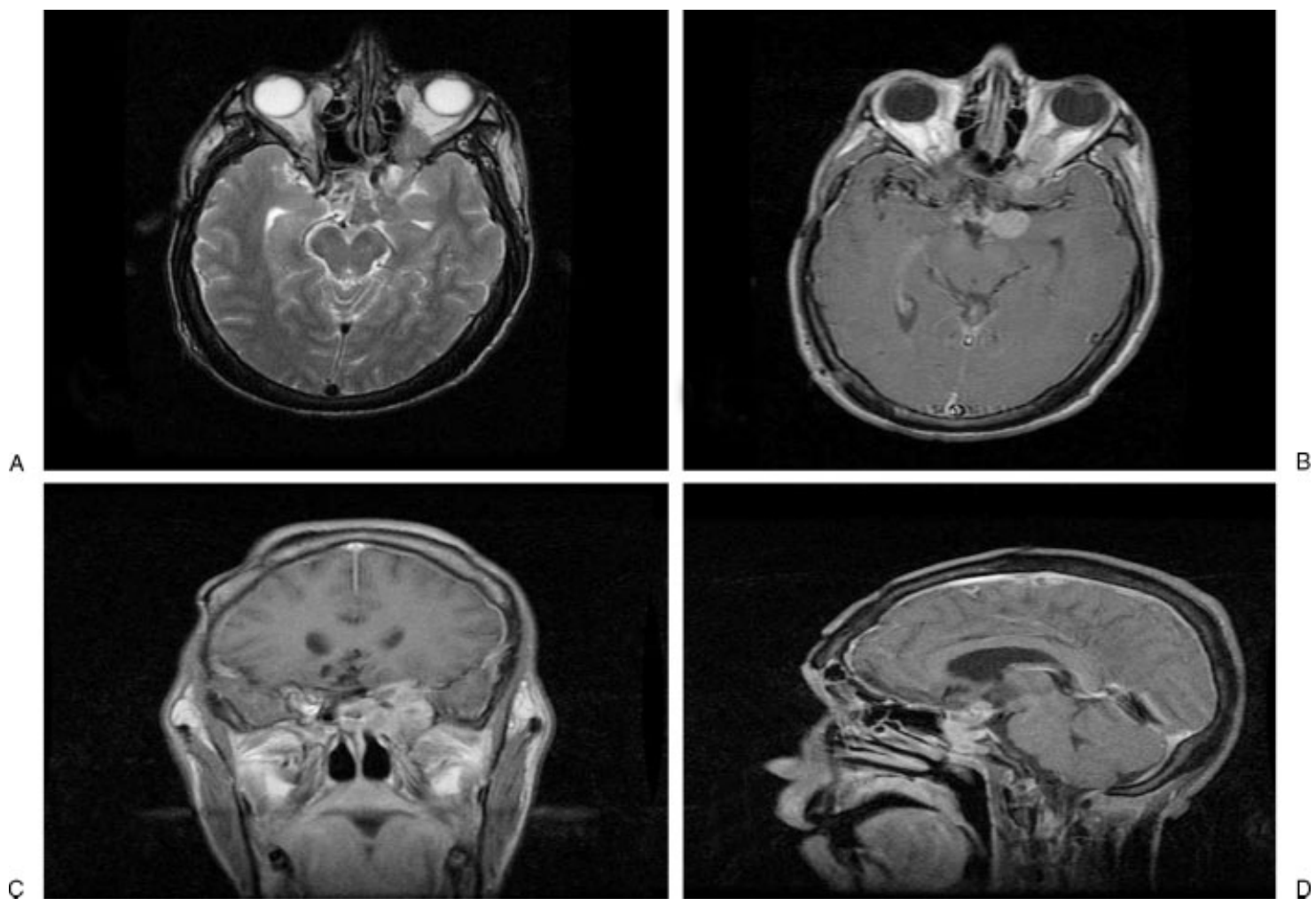

Figure 4 (A) Axial T1-weighted, (B) axial, (C) coronal, and (D) sagittal contrast images demonstrating the residual pituitary adenoma with a small fungal granuloma in the sphenoid sinus. 
Headache, impaired mentation, visual disturbances, extraocular muscle palsy, and endocrine failure are most common modes of presentation., 5,12 Other researchers have reported cases of primary pituitary Aspergillus abscess without any evidence of sinus infection. ${ }^{6-8}$ Among 21 patients of CNS aspergillosis reported by Murthy, 13 had skull base syndrome; 5 had cavernous sinus syndrome; orbital apex syndrome and cranial neuropathy were seen in 3 patients each, and proptosis in $2 .{ }^{12}$ Sunderam reported an incidence of $72 \%$ for skull base syndrome among 89 patients with CNS aspergillosis; of these patients, 8 had sino-orbital and 9 had sino-orbito-cranial involvement. ${ }^{12}$

Craniocerebral aspergillosis of sinonasal origin has typical MRI features of a mass lesion producing hypo- to isointense signals on T1-weighted images, hypointensity on $\mathrm{T} 2$-weighted images, with bright homogenous peripheral contrast enhancement. ${ }^{9}$ The clinical presentation and initial radiological tests in our acromegalic patient were confused with an apoplectic event causing panhypopituitarism in a setting of diabetic ketoacidosis. The possibility of a granuloma should be suspected whenever lipid and lactate peak are seen or MRS (Fig. 3b). Other differentiating features are the invasiveness of the fungal lesion and destruction of the surrounding bone on CT (Fig. 3a). Patients with diabetic ketoacidosis are at high risk of developing invasive rhinosinusoidal mycoses, especially mucormycosis. ${ }^{5}$

Surgery by transsphenoidal approach is preferred to transcranial route to avoid CSF contamination and meningitis. ${ }^{6,8,13,14}$ Meticulous clearance of the granuloma from the sphenoid sinus and sellar and nasal cavity, avoiding arachnoid breach in the sella, are vital for a good outcome. ${ }^{13}$ Amphotericin-B or combinations of amphotericin with flucytosine or itraconazole are the preferred drugs of choice. ${ }^{5,6,14}$ Liposomal amphotericin-B has proved to be very effective and safe in treating CNS aspergillosis. ${ }^{6}$ Nearly $30 \%$ of focal CNS aspergillosis can be cured with surgery and antifungal therapy. ${ }^{6}$ Cerebral aspergillosis carries a poor prognosis with a mortality of nearly $100 \%$ in immunocompromised patients and 40 to $80 \%$ in immunocompetent individuals. ${ }^{5,9,12,14}$

\section{CONCLUSION}

The presence of a dual pathology involving a granuloma in a patient operated for a pituitary adenoma can confuse the clinical presentation. Our case was unusual because the clinical picture was complicated by a preexisting pituitary adenoma, associated hormonal imbalances, and metabolic alterations due to erratic management of the diabetic state. Identification of a granulomatous pathology was possible based on previous history of transsphenoidal surgery, his uncontrolled diabetic status, MRI, and MRS. Sellar granulomas should be considered as a differential diagnosis of a pituitary lesion with atypical radiological features especially in immunocompromised individuals. Surgical decompression followed by antifungal chemotherapy provides optimal treatment.

\section{REFERENCES}

1. Chakrabarti A. Epidemiology of central nervous system mycoses. Neurol India 2007;55:191-197

2. Goel A, Nadkarni T, Desai AP. Aspergilloma in the paracavernous region-two case reports. Neurol Med Chir (Tokyo) 1996;36:733-736

3. Bhadada S, Bhansali A, Nahar U, et al. An unusual association of acromegaly and pituitary tuberculosis. Pituitary 2004;7:103-106

4. Sharma MC, Vaish S, Arora R, Gaikwad S, Sarkar C. Composite pituitary adenoma and intrasellar tuberculoma: report of a rare case. Pathol Oncol Res 2001;7:74-76

5. Dubey A, Patwardhan RV, Sampth S, Santosh V, Kolluri S, Nanda A. Intracranial fungal granuloma: analysis of 40 patients and review of the literature. Surg Neurol 2005; 63:254-260; discussion 260

6. Hao L, Jing C, Bowen C, Min H, Chao Y. Aspergillus sellar abscess: case report and review of the literature. Neurol India 2008;56:186-188

7. Iplikcioglu AC, Bek S, Bikmaz K, Ceylan D, Gökduman CA. Aspergillus pituitary abscess. Acta Neurochir (Wien) 2004;146:521-524

8. Ramos-Gabatin A, Jordan RM. Primary pituitary aspergillosis responding to transsphenoidal surgery and combined therapy with amphotericin-B and 5-fluorocytosine: case report. J Neurosurg 1981;54:839-841

9. Azarpira N, Esfandiari M, Bagheri MH, Rakei S, Salari S. Cerebral aspergillosis presenting as a mass lesion. Braz J Infect Dis 2008;12:349-351

10. Go JL, Kim PE, Ahmadi J, Seagall HD, Zee CS. Fungal infections of the central nervous system. Neuroimaging Clin N Am 2000;10:409-425

11. Endo T, Numagami Y, Jokura H, Ikeda H, Shirane R, Yoshimoto T. Aspergillus parasellar abscess mimicking radiation-induced neuropathy. Case report. Surg Neurol 2001;56: 195-200

12. Murthy JM. Fungal infections of the central nervous system: the clinical syndromes. Neurol India 2007;55:221-225

13. Gupta A, Gupta RK, Banerjee D, Bhatia E. Magnetic resonance image detection of coincidental sphenoid sinus aspergillosis and pituitary microadenoma: a potential surgical disaster. Australas Radiol 1998;42:128-129

14. Shamim MS, Siddiqui AA, Enam SA, Shah AA, Jooma R, Anwar S. Craniocerebral aspergillosis in immunocompetent hosts: surgical perspective. Neurol India 2007;55: 274-281 